\title{
Difusão do prontuário eletrônico do cidadão: estudo de caso em Minas Gerais
}

\author{
Dissemination of the Electronic Citizen's Record: a case study in Minas Gerais \\ Difusión del Registro Ciudadano Electrónico: estudio de caso en Minas Gerais
}

\section{RESUMO}

Objetivo: analisar processo de difusão do Prontuário Eletrônico do Cidadão no trabalho de equipes de saúde da família. Métodos: estudo de caso único holístico, de abordagem qualitativa, à luz da teoria de difusão da inovação. Dados coletados entre março e maio de 2021 , em sete equipes de saúde da família de um município de Minas Gerais. Foram entrevistados 31 profissionais de saúde e 3 informantes chave envolvidos com a implantação e utilização da tecnologia, aplicado questionário sociodemográfico e observações diretas do cotidiano de trabalho. A análise dos dados foi a partir da Análise de Conteúdo e com auxílio do software MAXQDA. Resultados: verificamos que a difusão da inovação avaliada apresentou vantagens em comparação com a tecnologia anteriormente utilizada no município, apresentando também (in)compatibilidades e complexidades em seu processo de difusão. Conclusão: a difusão do Prontuário Eletrônico do Cidadão depende de fortalecimento de sua compatibilidade ao trabalho e desenvolvimento da percepção de seu uso vantajoso.

DESCRITORES: Difusão de Inovações; Atenção Primária a Saúde; Prontuário Eletrônico; Sistema de Informação em Saúde; Informação em Saúde.

\section{ABSTRACT}

Objective:analyzed the process of dissemination of the Electronic Citizen's Record in the work of professionals from family health teams. Methods: a single holistic case study, with a qualitative approach, in the light of the innovation diffusion theory. Data collected between March and May 2021, in seven family health teams in a municipality in Minas Gerais. We interviewed 31 health professionals and 3 key informants involved with the implementation and use of technology, applied a sociodemographic questionnaire and carried out direct observations of daily work. Data analysis was based on Content Analysis and with the help of the MAXQDA software. we verified that the diffusion of the evaluated innovation presented advantages compared to the technology previously used in the city, also presenting (in)compatibilities and complexities in its diffusion process. and development of the perception of its beneficial use.

DESCRIPTORS: Diffusion of Innovations; Primary Health Care; Electronic Medical Record; Health Information System; Health Information

\section{RESUMEN}

Objetivo: analizar el proceso de difusión del Registro Ciudadano Electrónico en el trabajo de los profesionales de los equipos de salud de la familia. Métodos: estudio de caso único, holístico, con enfoque cualitativo, a la luz de la teoría de la difusión de la innovación. Datos recolectados entre marzo y mayo de 2021, en siete equipos de salud familiar de un municipio de Minas Gerais. Entrevistamos a 31 profesionales de la salud y 3 informantes clave involucrados con la implementación y uso de la tecnología, aplicamos un cuestionario sociodemográfico y realizamos observaciones directas del trabajo diario. El análisis de datos se basó en el análisis de contenido y con la ayuda del software MAXQDA. comprobamos que la difusión de la innovación evaluada presenta ventajas frente a la tecnología previamente utilizada en la ciudad, presentando también (in) compatibilidades y complejidades en su proceso de difusión. y desarrollo de la percepción de su uso beneficioso

DESCRIPTORES: Difusión de Innovaciones; Primeros auxilios; Historial médico electrónico; Sistema de información sanitaria; Información de salud

RECEBIDO EM: 08/06/21 APROVADO EM: 14/06/21

Talita Ingrid Magalhães Silva

Instituição: Doutoranda pela Universidade Federal de Minas Gerais - UFMG, Belo Horizonte, MG. Enfermeira ORCID: 0000-0003-0230-7439

\section{Thais Barreiros Tavares}

Mestranda na Universidade Federal de Juiz de Fora - Programa de Pós Graduação em Enfermagem, Juiz de Fora, MG . Enfermeira.

ORCID: 0000-0001-5076-4736

\section{Bruna de Souza Pena}

Mestranda no Programa de Pós Graduação em Enfermagem da Universidade Federal de Juiz de Fora, Juiz de Fora, MG . Enfermeira.

ORCID: 0000-0003-1938-2160 


\section{Regina Consolação Santos}

Doutorando no Programa de Pos Graduação em Psicologia da Universidade Federal de Juiz de Fora, MG. Enfermeira ORCID: 0000-0002-7393-3210

\section{Marilia Alves}

Prof. Dra ${ }^{a}$. Escola de Enfermagem/Programa de Pós Graduação em Enfermagem da Universidade Federal de Minas Gerais, Belo Horizonte, MG .Enfermeira.

ORCID: 0000-0002-4695-0787

\section{Tarcisio Laerte Gontijo}

Profo Adjunto Departamento de Enfermagem - Universidade Federal de São João del Rei, Divinópolis, MG. Enfermeiro.

ORCID: 0000-0001-7845-3825

\section{Ricardo Bezerra Cavalcante}

Prof $^{\circ}$. Adjunto da Escola de Enfermagem da Universidade Federal de Juiz de Fora; Profo. do Programa de Pós Graduação em Enfermagem da UFJF, MG . Enfermeiro.

ORCID: 0000-0001-5381-4815

\section{INTRODUÇÃO}

A Estratégia e-SUS Atenção Básica (e-SUS AB), lançada no ano de 2013, visa a informatização qualificada do Sistema Único de Saúde (SUS) em busca de um "SUS eletrônico"1. Para tanto, vale-se da implantação de inovações tecnológicas como o Prontuário Eletrônico do Cidadão (PEC), buscando o gerenciamento qualificado de informações advindas do processo de trabalho no contexto da Atenção Primária a Saúde (APS). O PEC é um software que opera como um repositório online onde informações clínicas e administrativas do paciente podem ser gerenciadas ${ }^{2}$.

A Estratégia e-SUS Atenção Básica (e-SUS AB), lançada no ano de 2013, é um recurso que atende ao processo de informatização qualificada do SUS em busca de um "SUS eletrônico"1,2. O Prontuário Eletrônico do Cidadão (PEC), como uma modalidade do e-SUS, é um software que opera como um repositório online onde todas as informações de saúde, clínicas e administrativas do paciente ficam armazenadas ${ }^{1,2}$. Estudo realizado na Região Sul do Brasil evidenciou que o uso do PE contribui para a gestão do cuidado, tomada de decisão e planejamento do trabalho do enfermeiro da $\mathrm{AB}^{3}$.

A incorporação do PEC representa possibilidades de qualificação e avanço no uso da informação na APS, e, além disso, impli-

\section{A Estratégia e-SUS}

Atenção Básica (e-SUS AB), lançada

no ano de 2013,

é um recurso que

atende ao processo

de informatização

qualificada do SUS

em busca de um

"SUS eletrônico" ca em mudanças estruturaisno ideário e na prática de profissionais e usuários dos serviços de saúde, conformando-se assim como uma inovaçãa $0^{3,5,6,7,8}$. Corroborando com esse pensamento, os recursos tecnológicos utilizados na Atenção Primária e Suplementar em Saúde ampliam e interligam seus princípios, buscando soluções resolutivas, qualificadas, práticas e acessíveis para as necessidades reais deste segmento ${ }^{4}$.

Entretanto, o PEC como uma inovação precisa ser avaliado no sentido de potencializar a sua adoção, seu aprimoramento e dos processos correlatos quando da implantação de tecnologias no cotidiano de trabalho em saúde. Sabe-se que a avaliação do processo de difusão de inovações é determinante para o sucesso ou fracasso de sua implantação e incorporação ao cotidiano8. Para tanto, a Teoria da Difusão de Inovações (TDI) é um importante referencial de norteamento da avaliação do processo de difusão de inovações?.

No Brasil, estudos conduzidos à luz da TDI instrumentalizaram investigações em diversas áreas do conhecimento, porém na saúde a sua utilização ainda é tímida quando comparada à utilização ao redor do mundo?. Assim, o processo de difusão de uma inovação é crucial e determinante para a sua adoção ou rejeição pelos indivíduos/ adotantes. Ainda que protocolos, tecnologias duras (software) e programas sejam apresentados aos profissionais de saúde, estes, é que decidem o que será de fato utili- 


\section{artigo}

Silva, T. I. M., Tavares, T. B., Pena, B. S., Santos, R. C., Alves, M., Gontijo, T. L.,Cavalcante, R. B.

Difusão do prontuário eletrônico do cidadão: estudo de caso em Minas Gerais

zado e o modo de utilização $0^{10,11,5,7}$.

Este estudo justifica-se pela necessidade de melhor compreender o processo de difusão do PEC como estratégia ministerial de âmbito nacional com repercussões no cotidiano de trabalho dos profissionais de saúde e na produção de informação em saúde na e para a AB. Parte-se do pressuposto de que o trabalho em saúde na $A B$ seja afetado pela incorporação de tecnologias duras como o PEC favorecendo percepções negativas a partir do uso do software, resistência a tecnologia e sua subutilização, bem como precarização das relações de trabalho.

Logo, a forma como o PEC tem sido difundido entre os profissionais de saúde pode gerar um ambiente favorável para que aspectos negativos sejam captados/ percebidos em detrimento dos positivos. A resistência a tecnologia, o modus operandi de sua utilização, suas repercussões e os desafios emergentes são aspectos complexos a serem compreendidos. Entretanto, para esta pesquisa, definiu-se como questão norteadora: Como tem ocorrido a difusão do
PEC no cotidiano de trabalho das equipes de saúde da família (eSF)?

Destarte, buscou-se analisar o processo de difusão do PEC no cotidiano de trabalho de profissionais de equipes de saúde $\mathrm{da}$ família à luz da Teoria da Difusão de Inovações.

\section{MÉTODO}

Trata-se de um estudo de caso único holístico, de abordagem qualitativa, a luz da TDI. Justifica-se o estudo de caso único devido a necessidade de investigar um fenômeno contemporâneo, a difusão de uma inovação (o PEC), em profundidade e em seu contexto real ${ }^{12}$. $\mathrm{O}$ uso da $\mathrm{TD}^{17}$ justifica-se pela necessidade de investigar o processo de difusão de uma inovação a partir de seus elementos e atributos, conforme descrito no Quadro 1.

$\mathrm{O}$ presente estudo pretende analisar a difusão do PEC no cotidiano de trabalho de equipes de saúde da família, e para tal, o cenário ideal para apreensão do fenômeno é onde o mesmo acontece ${ }^{12}$. Destaca-se que a definição do cenário se deu durante a pandemia de COVID-19, fato que restringiu o acesso aos municípios em atendimento às restrições recomendadas pelo Programa Minas Consciente ${ }^{13}$. Entretanto, após as devidas aprovações pelo município e respeitando os protocolos sanitários, optou-se por manter a coleta presencial devido a necessidade de apreender a percepção dos adotantes associada a observação de aspectos no cotidiano de trabalho não captáveis apenas pela entrevista.

Neste interim, a coleta de dados foi realizada entre os meses de abril a junho de 2021, em um município da Região Ampliada de Saúde - Oeste de Minas Gerais, escolhido intencionalmente, que denominamos como "A" para garantir o anonimato. Os participantes do estudo foram 31 profissionais de saúde da eSF (médicos, enfermeiros e agentes comunitários de saúde - ACS) de acordo com os seguintes critérios de inclusão: ser profissional da eSF; estar utilizando PEC no período da coleta de dados; ter

\section{Quadro 1 -Elementos e Atributos da Teoria da Difusão de Inovações}

\begin{tabular}{|c|c|}
\hline ELEMENTOS & \\
\hline $\begin{array}{l}\text { Inovação: (é uma } \\
\text { ideia ou prática } \\
\text { percebida como } \\
\text { nova por adotan- } \\
\text { tes futuros) }\end{array}$ & ATRIBUTOS \\
\hline $\begin{array}{l}\text { Canais de } \\
\text { Comunicação }\end{array}$ & $\begin{array}{l}\text { Conceito: Canais em que as mensagens/ } \\
\text { informações sobre a inovação circulam } \\
\text { entre indivíduos ou grupos de um } \\
\text { mesmo sistema social, considerando a } \\
\text { sua natureza. }\end{array}$ \\
\hline Tempo & $\begin{array}{l}\text { Conceito: A dimensão do tempo entre o } \\
\text { contato com a inovação e a decisão de } \\
\text { aceitar ou rejeitar. }\end{array}$ \\
\hline Sistema Social & $\begin{array}{l}\text { Conceito: Unidades inter-relacionadas } \\
\text { com objetivos semelhantes. Os mem- } \\
\text { bros ou unidade de um sistema social } \\
\text { podem ser individuais, grupos informais } \\
\text { ou organizações. }\end{array}$ \\
\hline
\end{tabular}

Fonte: Elaborado pelos autores, 2021

Vantagem Relativa: Percepção de que o uso da inovação é vantajoso, melhor do que as práticas anteriores Compatibilidade: Percepção de que a inovação é consistente com os valores, experiências passadas e necessidades dos adotantes.

Complexidade: Percepção de que o uso e a compreensão da inovação são considerados difícil.

Experimentação: Período em que a inovação é experimentada/testada antes de sua implantação efetiva.

Observabilidade: Percepções sobre os resultados, efeitos da inovação visíveis no cotidiano. 
mais de 06 meses de utilização do PEC para registro do atendimento à população. Foi critério de exclusão estar ausente por férias ou licença médica no período de coleta de dados. No decorrer do estudo, foram incluídos 3 informantes chave da Secretaria Municipal de Saúde, detentores de informações estratégicas para a implantação/ operacionalização do PEC. Assim,este estudo contou com 34 participantes ao total.

Os dados foram obtidos através de questionário para caracterização dos participantes, entrevista dirigida por roteiro semiestruturado e observação direta no cenário de estudo. As variáveis do questionário foram: profissão, cargo ocupado, idade, escolaridade, graduação, tempo de trabalho na APS. Os dados coletados a partir das entrevistas e observações foram submetidos a Análise de Conteúdo, modalidade Temático-Categorial, a partir de três fases: pré-análise; exploração do material e o tratamento dos resultados; inferência e interpretação ${ }^{14,15}$.

$\mathrm{Na}$ pré-análise, foi realizada leitura flutuante de todo o material no primeiro contato com o texto a ser analisado e um alinhamento mais preciso com os objetivos do estudo (entrevistas e diário de campo) organização do material e preparo para transformar os dados brutos em material de análise. Os códigos foram reagrupados de acordo com a temática a que se referiam, elencando as temáticas identificadas, construindo as categorias, que foram analisadas por meio de formulação das inferências e interpretações derivadas principalmente das captações do pesquisador.

Os procedimentos metodológicos adotados neste estudo atenderam as Resoluções do Conselho Nacional de Saúde $510 / 2016$ e $262 / 2012$. A pesquisa foi aprovada pelo Comitê de Ética em Pesquisa com Seres Humanos da Universidade Federal de Minas Gerais - parecer n ${ }^{\circ} 4.770 .276$ e Universidade Federal de São João Del Rei - parecer no 4.662.362. O Termo de Consentimento Livre e Esclarecido (TCLE) por escrito foi aplicado em todos os participantes (entrevistados e observados diretamente), garantiu-se o sigilo, o anonimato e a privacidadedos participantes.

\section{RESULTADOS E DISCUSSÕES}

O perfil dos participantes revelou a predominância do gênero feminino com $91,18 \%(n=31)$ e $70,59 \%(n=24)$ são con-

\section{A incorporação do \\ PEC no cotidiano}

das eSF não

representou até

o momento, uma

ruptura completa

com as outras

formas de registro

em papel usuais

nas unidades, e

que de certa forma

proporcionam o

desenvolvimento

da percepção da

necessidade de

\section{continuidade de seu}

uso

cursados em regime estatutário. $\mathrm{O}$ tempo médio de trabalho na área da saúde foi de 10,79 anos e o tempo médio de experiência em unidade de saúde da família foi de 6,13 anos. Em relação a carga horária de traba- lho, 94,12\% (32) declararam vínculo de 40 horas semanais com a eSF.

A difusão do PEC favorecida pela percepção devantagem relativa e observabilidade

No cenário em estudo, anteriormente à implantação do PEC, já havia outro sistema de informação, o "VIVER", uma tecnologia terceirizada, utilizada para realizar a gestão das informações advindas do cotidiano de trabalho:

"em outubro de 2019, veio uma equipe para cá, pra poder fazer a transição, né? Do VIVER para o e-SUS, PEC (...) E ai a gente migrou [referindo-se ao PEC]." (E09ENF02).

Para os profissionais: “(...) no VIVER faltava muita coisa.

A gente está arrumando os cadastros,está bem melhor"(E18ACSO3).

Outro entrevistado endossa a vantagem de uso do PEC:

“(...) eu acho que melhorou em relação a antes, quando era o VIVER.

Eu gosto dessa proposta nova do PEC (...) eu acho que é um ganho sim o prontuário eletrônico." (E01ENF01).

Entretanto, a incorporação do PEC no cotidiano das eSF não representou até o momento, uma ruptura completa com as outras formas de registro em papel usuais nas unidades, e que de certa forma proporcionam o desenvolvimento da percepção da necessidade de continuidade de seu uso. Nesse sentido reconhece-se que é mais vantajoso continuar utilizando o papel em paralelo ao uso da tecnologia (o PEC), mesmo que o artefato tecnológico represente uma inovação. Um dos entrevistados explica o uso do PEC concomitante ao uso do papel: 
"Por conta das inconsistências, pela facilidade as vezes que o sistema está fora do ar, as vezes você precisa consultar alguma coisa... eu prefiro manter os dois" (E09ENF02).

Outro entrevistado também reconhe-

\section{“Às vezes atendia} o paciente e ficava aquela pilha de prontuário para evoluir. Agora com o PEC eu tento me esforçar para já fazer aquele atendimento na hora” (E01ENF01)

ceu:

"Eu comecei com o papel,mas depois, eu fiz o esforço e me adaptei (...) agora eu já estou integrado (...) agora eu já vou sozinho [referindo-se ao uso do PEC]. (E03MED01)

Para Rogers ${ }^{7}$ a aceitação de uma inovação depende do desenvolvimento da per- cepção de que há uma vantagem relativa em usá-la em detrimento da anterior. Assim, a necessidade de um novo prontuário eletrônico foi reconhecida devido ao fato do VIVER apresentar inconsistências e não ser mais compatível com a realidade de trabalho dos profissionais. Entretanto, a maximização da aceitação do PEC depende do desenvolvimento de uma percepção de que o seu uso pode ser independente do registro em papel. A implantação do PEC em todo o país tem como propósito favorecer a substituição completa do papel para registro das informações no cotidiano de trabalho das $\mathrm{eSF}^{16,17}$. Além disso, sabe-se que o uso do papel para registro das informações no contexto das organizações de saúde é uma prática que não está totalmente alinhada a segurança do paciente e as dificuldades relacionadas ao planejamento local e a tomada de decisões qualificadas ${ }^{18,19}$.

Os entrevistados observaram benefícios da adoção do PEC (observabilidade) no cotidiano de trabalho, o que fortaleceu o desenvolvimento da percepção da vantagem relativa do $\mathrm{PEC}$ em relação à tecnologia anterior. Reconheceu-se que o PEC beneficia a sistematização do registro das informações:

"Às vezes atendia o paciente e ficava aquela pilha de prontuário para evoluir. Agora com o PEC eu tento me esforçar para já fazer aquele atendimento na hora" (E01ENF01).

“(...) eu acho muito completo, você quer um atestado, uma prescrição, lista dos medicamentos, atestado de comparecimento, um encaminhamento. Tudo está aqui." (E07DEN01).

Como destacado por Rogers ${ }^{7}$, os possíveis adotantes de uma inovação precisam observar resultados positivos a partir de seu uso. Isso é essencial para a difusão qualificada de uma inovação. Não basta apenas perceber que a inovação atual é vantajosa em relação à anterior, é preciso que suas contribuições para o trabalho sejam aparentes e com transformações sobre as práticas cotidianas ${ }^{9}$.

As (in)compatibilidades, complexidades e canais de comunicação no sistema social influenciando a difusão da inovação

Alguns entrevistados destacaram a necessidade de sintetizar algumas informações no PEC, alegando ser extenso o seu preenchimento, o que remete à parte de suas (in) compatibilidades e complexidades:

"eu mudaria em relação a estruturação da anamnese, eu sei que usaram o método SOAP. Está muito extenso (...) demanda tempo, difícil de usar" (E08MED02).

A inovação também poderia ter aprimorado o seu processo de recuperação da informação:

"O sistema precisa melhorar nesse
sentido da gente conseguir monito-
rar o que fez através de relatórios,
mais filtros, no sentido de pesquisar
as coisas" (EO1ENF01).

Além da necessidade de integração das informações entre os pontos da rede assistencial:

"você lança aqui, manda pro pronto socorro, e eles não mandam a contra referência no sistema" (E03MED01).

A necessidade de otimizar o tempo e a sobrecarga de trabalho são situações que justificam, na perspectiva dos profissionais, as adaptações no uso do PEC, minimizando a complexidade de sua utilização. Em relação ao médico, por exemplo, observamos a organização de sua agenda para o atendimento de 14 consultas no período da manhã e outras 14 no período da tarde. Deste modo, em 8 horas diárias de trabalho, o profissional médico deve atender 28 usuários, e com posterior registro no prontuário físico e eletrônico [NO]. 
Isso dificulta o registro imediato dos atendimentos, tal dinâmica (principalmente de médicos e enfermeiros) encontra no PEC outras possibilidades de registro, no chamado "registro tardio" (E01ENF01).

Também verificamos profissionais, que por hábito, mantém práticas que podem se conformar como dificultadores da adoção da inovação aumentando a complexidade de sua adoção:

"eu registro tudo no prontuário fisico porque eu não perdi o hábito(...) eu sou muito apegado as coisas antigas. Eu registro no prontuário eletrônico só no final do expediente. (E12MED03)".

Em uma cena observamos um médico e duas enfermeiras confessando registrar "apenas alguns" dos atendimentos, além de optar por "colocar" no sistema "apenas o necessário", isso devido à sobrecarga de trabalho. Entendem que o PEC ainda é uma obrigatoriedade, sua adoção é controversa e complexa[NO].

A compatibilidade para Rogers ${ }^{7}$ está relacionada ao significado atribuído ao trabalho e as particularidades singulares de quem o executa. Ainda que a incorporação tecnológica esteja à primeira vista, relacionada à padronização e necessidade da captura do trabalho, o processo de difusão de uma inovação vai, além disso. A singularidade de cada profissional deve ser considerada no processo de difusão através da compatibilidade da inovação às necessidades do adotante, remetendo ao caráter dinâmico e flexível que acolhe a práxis do trabalhador/ adotante. A compatibilidade neste sentido está relacionada a (re)ssignificação da inovação tornando-a menos complexa para o adotante. Neste sentido, a complexidade oscila entre presença e ausência, que pode ser descrito como um vai e vem de movimentos entre os atores envolvidos. Assim, as dificuldades percebidas vão sendo superadas e à medida que o adotante se apropria da inovação?.

Como descrito por Rogers7, há desafios específicos na difusão de inovações relacionadas a softwares pelo caráter imaterial que os envolve, entretanto, o uso de meios de divulgação/comunicação em massa pode favorecer a familiaridade e o sucesso da adoção. Emergiu nos discursos, o reconhecimento destes canais de comunicação como elementos de contribuição a adoção do PEC, diminuindo suas incompatibilidades e mitigando suas complexidades:

"eu entrei no tutorial do PEC pra ver como que a gente preenchia (...) tirou as minhas dúvidas e tudo. Porque as vezes eu não preenchia todos os tópicos que tinha pra preencher." (E07DEN01).

Assim, a acessibilidade das informações sobre o PEC, esteve atrelada à mobilização entre as pessoas, por meio de canais de comunicação, no sistema social, favorecendo a difusão no sentido de mitigar suas complexidades. Os canais de comunicação determinam o modo como as informações são recebidas pelos adotantes e como elas circulam entre os demais envolvidos, auxiliando na persuasão e decisão para a aceitação da tecnologia $a^{20,21,7}$.

\section{CONCLUSÃO}

Buscamos neste estudo analisar o processo de difusão do PEC no cotidiano de trabalho de profissionais de equipes de saúde da família a luz da Teoria da Difusão de Inovações. Verificamos que a difusão do PEC é favorecida pelo desenvolvimento da percepção de suas vantagens em detrimento da tecnologia anterior, mas também como uma inovação que já possibilita a observação de resultados sobre o cotidiano de trabalho, aprimorando principalmente a gestão das informações no contexto da APS. (In) compatibilidades e complexidades também se fizeram presentes na difusão do PEC, porém foram minimizados a partir dos canais de comunicação existentes no sistema social constituído pela mobilização de atores que de alguma forma influenciaram a difusão da inovação.Como limitações deste estudo destacamos o contexto em que os dados foram coletados, o período da pandemia de COVID-19, influenciando o tempo de observação da pesquisadora nas unidades, bem como o desenvolvimento de algumas entrevistas.

Buscamos neste

estudo analisar

o processo de

difusão do PEC

no cotidiano

de trabalho de

profissionais de

equipes de saúde

da família a luz da

Teoria da Difusão

de Inovaçôes.

Verificamos que

a difusão do PEC

é favorecida pelo

desenvolvimento

da percepção de

suas vantagens

em detrimento da

tecnologia anterior

[...] 


\section{REFERÊNCIAS}

1. Brasil. Ministério da Saúde. Secretaria de Atenção à Saúde. Departamento de Atenção Básica. e-SUS Atenção Básica: manual de implantação. Brasília, DF. 2014c. 63 p.

2. Brasil. Ministério da Saúde. Secretaria de Atenção à Saúde. Departamento de Atenção Básica. Diretrizes nacionais de implantação da estratégia e-SUS AB. Brasília, DF, 2014b. 11 p.

3. Godoy JSM et al. The use of eletronic health records by nurses in Primary Health Units in Brazil. J. Health Inform. v. 4, n. 1, p. 3-9, 2012.

4. Silva BAG, Castro TMS, Venturi K. Gerenciamento do Enfermeiro em e-Saúde e a articulação dos princípios da Atenção Primária em Saúde e Saúde Suplementar: Relato de Experiência durante o início da Pandemia Covid-19. Revista Saúde Coletiva (10) n 53, 2020, 2348-2351.

5. Oliveira AEC et al. Implantação do e-SUS AB no Distrito Sanitário IV de João Pessoa (PB): relato de experiência. Saúde Debate, Rio de Janeiro, v. 40, n. 109, p. 212-218, abr./jun. 2016.

6. Brasil. Ministério da Saúde. Secretaria de Atenção à Saúde. Departamento de Atenção Básica. e-SUS Atenção Básica: Manual do Sistema com Prontuário Eletrônico do Cidadão PEC - Versão 3.1 [recurso eletrônico] / Ministério da Saúde, Secretaria de Atenção à Saúde, Secretaria-Executiva. - Brasília: Ministério da Saúde, 2018. 466 p.

7. Rogers EM. Diffusion of innovation. 5rd ed. New York: The Free Press, 2003. $551 \mathrm{p}$.

8. Silva TIM et. al. Diffusion of the e-SUS Primary Care innovation in Family Health Teams. Rev Bras Enferm [Internet]. v. 71, n. 6, p. 2945-52. 2018. DOl: http://dx.doi.org/10.1590/0034-7167-20180053

9. Rago CAP, Zucchi P. Prontuário Eletrônico do Paciente: como a teoria da difusão de inovações pode colaborar na sua implantação. J. Health Inform., v. 9, n. 2., p. 57-61, abr./jun. 2017.

10. Cabral SAAO et al. A utilização do e-SUS (AB) no processo de fortalecimento da efetivação dos princípios doutrinários do SUS. INTESA, Rio de Janeiro, v. 9, n. 1, p. 1-4, 2015.

11. Holmes ES et al. Health information systems in the decision-making process in primary care. International Archives of Medicine Section: Global Health \& Health Policy, London, v. 9, n. 2, Jan. 2016. Disponivel em: <http://imed.pub/ojs/index.php/iam/article/ view/1380>. Acesso em: 10 dez. 2016.

12. Yin R. Estudo de caso: planejamento e métodos. 5ed. Porto Alegre: Bookman; 2015.

13. Minas Gerais. Secretaria de Estado da Saúde de Minas Gerais. Secretaria de Desenvolvimento Econômico. Minas Consciente: Retomando a economia do jeito certo. Protocolo versão 3.9 19/07/2021. 21p. 2021c. Disponível em: www.mg.gov.br/sites/default/files/paginas/imagens/minas

14. Fontanella BJB et al. Sampling in qualitative research: a proposal for procedures to detect theoretical saturation. Cad. Saúde Pública, Rio de Janeiro, v. 27, n. 2, p. 389-394, 2011. Disponivel em: http:// www.scielo.br/pdf/csp/v27n2/20.pdf

15. Bardin L. Análise de conteúdo. 4. ed. Lisboa: Ed. 70, 2015. 288 p.

16. Brasil. Ministério da Saúde. Secretaria de Atenção à Saúde. Departamento de Atenção Básica. e-SUS Atenção Básica: Manual do Sistema com Prontuário Eletrônico do Cidadão PEC - Versão 3.1 [recurso eletrônico] / Ministério da Saúde, Secretaria de Atenção à Saúde, Secretaria-Executiva. - Brasília: Ministério da Saúde, 2018. $466 \mathrm{p}$.

17. Ifinedo P. Empirical study of Nova Scotia Nurses' Adoption of Healthcare Information Systems: Implications for Management and Policy-Making. Int. J. Health Policy Manag. [Internet], v. 7, n. 4: p. 317-327. 2018 DOI: doi10.15171/ijhpm.2017.96

18. Thum M, Baldisserotto J, Celeste RK. e-SUS AB use and factors associated with recording primary care procedures and consultations in Brazilian municipalities. Cad. Saúde Pública 2019; 35(2):e00029418. doi:10.1590/0102-311X00029418

19. Pinheiro ALS et al. Gestão da saúde: o uso de sistemas de informação e o compartilhamento de conhecimento para a tomada de decisão. Texto e Contexto Enferm. Florianópolis, v. 25, n. 3, p. 1 - 9, 2016. Acesso em: 07 jan 2017. DOI: http://dx.doi.org/10.1590/010407072016003440015

20. Taherdoost $\mathrm{H}$. A review of technology acceptance and adoption models and theories. Procedia Manufacturing [Internet]. v. 22, p. 960-967. 2018. DOI: 10.1016/j.promfg.2018.03.137

21. Rogers EM. Diffusion of innovation. 3rd ed. New York: The Free Press, 1983. $452 \mathrm{p}$. 